\title{
Akzeptanzanalyse zum Einsatz von Hybriden Prototypen und Extended Reality in der Produktentstehung
}

Liesa Zimmermann, Kathrin Konkol, Elisabeth Brandenburg, Rainer Stark

Anhand einer Befragung zu virtuellen und hybriden Prototypen, einem multimodalen Lösungsansatz zur Beurteilung von virtuellen Prototypen (Buchholz 2017), werden Erwartungshaltungen, Erfahrungswerte und Prioritäten von Experten in der Produktentwicklung dargestellt und analysiert. Die qualitative Expertenbefragung spiegelt ein aktuelles Meinungsbild der deutschen Industrie zur Verwendung solcher Prototypen wieder und eröffnet eine detaillierte Sicht auf Hürden, die es bei der Entwicklung und Planung zu bewältigen gilt, um die Hemmschwellen für den Einsatz mindern zu können. Im Zuge des Verbundprojekts "RobVRAR - Funktionale virtuelle Prüfsysteme für die roboterbasierte Qualitätsprüfung" ist in Zusammenarbeit mit der Firma Battenberg ROBOTIC GmbH \& Co. KG ein Heckklappen-Demonstrator gebaut worden, der sich als repräsentatives Beispiel eignet. Die Expertenbefragung enthält allgemeine Fragen zu hybriden Prototypen und Fragen, die sich konkret mit dem Heckklappen-Demonstrator befassen.

Extended Reality, Hybride Prototypen, Expertenbefragung

\section{Hybride Prototypen in der virtuellen Produktentstehung}

In der Produktentstehung wird die Visualisierung virtueller Inhalte immer häufiger eingesetzt, um eine Beurteilung des aktuellen Entwicklungsstandes zu ermöglichen (Stark et al. 2009). Es kommen in diesem Kontext nicht nur herkömmliche Desktopanwendungen für die Visualisierung von 3D-Informationen zum Einsatz, sondern vermehrt auch Technologien wie Augmented Reality (AR) und Virtual Reality (VR). Studien deuten darauf hin, dass der Einsatz von VR in der virtuellen Inbetriebnahme zu einer intuitiven Bedienung auch für unerfahrene Nutzergruppen führt (Metzner et al. 2020). Im Kontext von Anlagenprozessen werden kritische Montage- und Handhabungsvorgänge des Werkers virtuell abgebildet und können so frühzeitig und kosteneffizient optimiert werden (Becker et al. 2018). Diese Prozesse werden nicht nur im virtuellen Raum abgebildet, sondern können weiterhin um haptische Elemente ergänzt werden. Diese Technologie, in der manuelle Aufgaben und Sachverhalte der Ingenieurstätigkeit im 
virtuellen Raum abgebildet und um haptische Komponenten ergänzt werden, nennt man hybride Prototypen. Der Einsatz von haptischen Reizen wird unter anderem auch im Bereich AR im Kontext der mobilen Geräte und tragbaren Geräten untersucht, wo zwar eine Vielzahl an vorhandenen Geräten festgestellt, allerdings hohe Kosten und fehlende Ortsunabhängigkeit der vorhandenen Lösungen bemängelt wird. Im Einsatz wird zusammenfassend eine verbesserte User Experience erreicht (Bermejo und Hui 2017). Weiterhin wurde anhand einer Studie in VR gezeigt, dass haptisches Feedback einen positiven Einfluss auf die Dauer von Designentscheidungen im virtuellen Raum haben kann (Volkov und Vance 2001). In einem Vergleich drei immersiver und haptischer Montagesimulation mit hybriden Prototypen in VR stellen (Grajewski et al. 2015) fest, dass der Einsatz von taktilem Feedback die Möglichkeit bietet, die Ergonomie eines Arbeitsplatzes zu überprüfen und Schulungen durchzuführen, allerdings mindert die eingeschränkte Mobilität des Demonstrators die Immersion stark.

In Zusammenarbeit mit der Firma Battenberg ROBOTIC GmbH \& Co. KG wurde im Rahmen des Projekts RobVRAR ein hybrider Prototyp entwickelt. Dieser stellt einen Demonstrator dar, der die Haptik einer Heckklappe eines Fahrzeugs simuliert, Interaktionsmöglichkeiten schafft und diese in Echtzeit in Virtual Reality anzeigt. Dazu wird das Design des Fahrzeugs und der Heckklappe virtuell über ein Head Mounted Display (HMD) ausgegeben, während das mechanische Verhalten anhand der kinematischen und kinetischen Größen simuliert und über einen Roboterarm ausgegeben und erlebbar gemacht wird. Am Tool Center Point des Roboterarms wird der Ausschnitt einer Heckklappe befestigt, der zum Öffnen und Schließen berührt wird, um ein realistisches Gefühl der Form und Oberflächenstruktur zu erreichen (siehe Abbildung 1).

\section{Faktoren für den Einsatz hybrider Prototypen}

Die Nutzerakzeptanz wird durch die beiden Faktoren Nützlichkeit und Zufriedenstellung definiert (van der Laan et al. 1997). Diese finden sich auch in den Ergonomieanforderungen für interaktive Systeme wieder, welche die Gebrauchstauglichkeit (Usability) definieren: Damit mit einem System effektiv (die Arbeitsaufgabe kann erfüllt werden), effizient (Aufgabenerfüllung in möglichst kurzer Zeit) und zufriedenstellend (der Nutzer empfindet keine Über- oder Unterbeanspruchung und hat Spaß an der Nutzung) gearbeitet werden kann (DIN EN ISO 9241-11).

Inwieweit diese Anforderungen bei der Nutzung von hybriden Prototypen erfüllt werden, ist nicht untersucht. Richtlinien zur Gestaltung von virtuellen Anwendungen ohne haptische Komponente hingegen schon (Brandenburg 2020; Bowman 2005). Insbesondere eine qualitativere Ausführung der Aufgabe in den Bereichen Design und Mon- 
tage wird belegt. Neben diesen Faktoren des virtuellen Anteils des hybriden Prototypen existieren grundlegende Schutzprinzipien für die Mensch-Roboter-Kollaboration: Sicherheitsgerichteter überwachter Stillstand bei Betreten des Mitarbeitenden in den gemeinsamen Arbeitsraum, Geschwindigkeits- und Abstandsüberwachung von Mitarbeitendem und in Bewegung befindlichen Roboter und Leistungs- und Kraftbegrenzung des Roboters auf ein ungefährliches Maß (ISO/TS 15066).

Da sich die Akzeptanz für die Anschaffung von neuen Technologien nicht nur auf den Endnutzer bezieht, werden alle Elemente der Mensch-Technik-Interaktion (Organisation, Mensch, Technik, Aufgabe) (DIN EN ISO 9241-11) herangezogen. Die Zusammenstellung der Faktoren für den Einsatz hybrider Prototypen ist in Tabelle zu sehen.

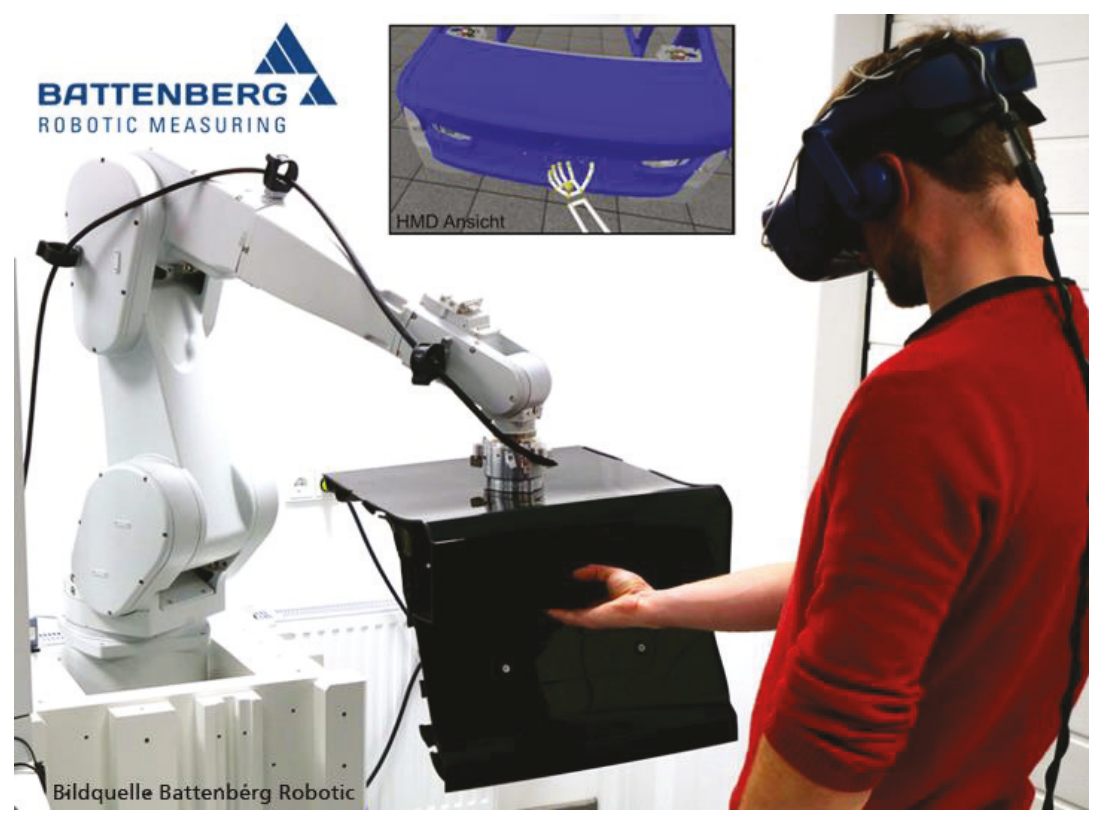

Abbildung 1: hybrider Prototyp Heckklappendemonstrator bei der Nutzung, Nutzer-Sicht durch das Head Mounted Display (oben) 
Tabelle 1: Zusammenfassung Faktoren für den Einsatz hybrider Prototypen

\begin{tabular}{|c|c|c|}
\hline \multirow[t]{4}{*}{ Organisation } & Kosten & beschreiben den Produktpreis bei der Anschaffung \\
\hline & Ortsunabhängigkeit & $\begin{array}{l}\text { beschreibt die Transportierbarkeit des Systems/ die Fä- } \\
\text { higkeit zum Remote Arbeiten }\end{array}$ \\
\hline & Umbauaufwand & $\begin{array}{l}\text { beschreibt die nötigen Mittel (Kosten, Zeit) zur Anpassung } \\
\text { an neuen Nutzungskontext }\end{array}$ \\
\hline & Innovation & beschreibt die Anwendung neuer Technologien \\
\hline \multirow[t]{4}{*}{ Mensch } & Sicherheit & $\begin{array}{l}\text { beschreibt das Potential der Gesundheitsgefährdung bei } \\
\text { der Nutzung }\end{array}$ \\
\hline & Ergonomie & $\begin{array}{l}\text { beschreibt die Anpassung der Arbeitsbedingungen an } \\
\text { den Nutzer }\end{array}$ \\
\hline & $\begin{array}{l}\text { Schulungsnotwendig- } \\
\text { keit }\end{array}$ & $\begin{array}{l}\text { beschreibt die Voraussetzung an Vorwissen/ Vorerfah- } \\
\text { rung/ Training des Nutzers }\end{array}$ \\
\hline & Spaß an der Nutzung & beschreibt die Benutzerfreundlichkeit \\
\hline \multirow[t]{2}{*}{ Technik } & Wartungsaufwand & beschreibt die nötigen Aufgaben zur Instandhaltung \\
\hline & Größe & $\begin{array}{l}\text { beschreibt die Außenmaße des Systems oder Arbeitsrau- } \\
\text { mes }\end{array}$ \\
\hline \multirow[t]{2}{*}{ Aufgabe } & Zeitersparnis & beschreibt die Effizienz \\
\hline & Modularität & $\begin{array}{l}\text { beschreibt das Potential zur Anpassung an einen neuen } \\
\text { Nutzungskontext }\end{array}$ \\
\hline
\end{tabular}

\section{Fragestellung}

Um die Akzeptanz der Industrie am Heckklappen-Demonstrator und ähnlicher Technologie besser abschätzen zu können, wurden Befragungen mit Experten verschiedener Branchen wie der Automobil- und der Möbelindustrie durchgeführt. So können die Chancen und Hemmschwellen zur Nutzung von hybriden und virtuellen Prototypen in der Industrie gefunden und adressiert werden. Aus der Expertenbefragung werden konkrete Anforderungen der Industrie an hybride Prototypen sowie die Schwierigkeiten bei der Einführung und Nutzung dieser neuen Technologie sichtbar. Eine solche Befragung liefert eine aktuelle Repräsentation der Meinung von Ingenieuren zu hybriden Prototypen im Verhältnis zu ihrer Erfahrung mit diesen Technologien. Zudem entstehen aus dem Format des offenen Gesprächs Ideen für weitere Anwendungsgebiete von hybriden Prototypen und konkret des Heckklappen-Demonstrators. 


\section{Expertenbefragung}

\section{Stichprobe}

Es wurden zehn Ingenieur*innen interviewt (davon eine weiblich). Drei Befragte sind in der Automobilindustrie tätig, drei in der Möbelindustrie und zwei im Maschinenbau. Sechs von diesen Unternehmen sind KMU mit bis zu 50 Mitarbeitenden. Vier Unternehmen sind Großunternehmen mit bis zu 300.000 Mitarbeitenden. Die Berufserfahrung der Befragten lag zwischen ein und 25 Jahren ( $M W=12,42$ und SD = 12,76). Sechs Personen gaben an, Erfahrung mit Augmented Reality zu haben, sieben Personen arbeiteten bereits mit Virtual Reality und zwei der befragten Personen arbeiteten mit hybriden Prototypen. Sechs der Befragten haben berufliche Erfahrung in der Robotik. Zwei der Befragten haben keinerlei Erfahrung mit den genannten Technologien. Die Hälfte der Probanden kommen arbeitsbedingt mit den Technologien AR und VR in Kontakt. Die jeweilige Erfahrung der Befragten erstrecken sich über diverse Themen der Industrie 4.0 - von der Qualitätsprüfung, Ergonomie-Prüfung und Digitalen Zwillingen zu Einbausimulationen und der Virtuellen Inbetriebnahme. In einem Fall wurde die Technologie für reine Demonstrationszwecke eingesetzt.

\section{Durchführung und Material}

Vorab wird den Befragten ein Video des hybriden Prototyps der Firma Battenberg sowie eine Beschreibung des Projektes zur Verfügung gestellt. Das Interview fand über die Plattform Cisco Webex Meetings statt. Nach einer kurzen Begrüßung und der positiv beantworteten Frage nach der Erlaubnis zum Audiomitschnitt, wurde der Mitschnitt gestartet und das Interview begonnen. Nach einer kurzen eigenen Vorstellung der Interviewpartner (Name, Beruf und Berufserfahrung) wurde der Fragebogen zu den demografischen Angaben auf der online-Plattform LamaPoll ausgefüllt. Anschließend wurde der Kontext des Projekts RobVRAR beschrieben, das Video gemeinsam betrachtet und offene Fragen geklärt, sowie ein erstes Feedback zu hybriden Systemen eingeholt. Danach wurde eine Bewertung der Faktoren zum Einsatz hybrider Prototypen vorgenommen. Die Faktorenbewertung erfolgte anhand einer fünfstufigen LikertSkala mit verbaler Verankerung ( 1 = niedrige Priorität bis 5 = hohe Priorität). Abschließend wurden offene Fragen gestellt, die sich auf den Einsatz der hybriden Prototypen beziehen und von den Autoren kategorisiert und deskriptiv ausgewertet wurden. Während der Interviews wurden die Gespräche aufgenommen und anschließend transkribiert. 


\section{Studienergebnisse}

\section{Auswertung Meinungsbild hybrider Prototyp Heckklappen-Demonstrator}

Das Meinungsbild ist positiv geprägt. Die Möglichkeit, haptisches Feedback aus einer Simulation zu erhalten stellt einen großen positiven Aspekt dar. Auch wurde die Sensibilität in der Führung der Klappe und die Integration von Handtracking positiv bewertet. Im Bereich Wirtschaftlichkeit sticht der geringe Platzbedarf, sowie schnell änderbare Simulationsparameter oder Hardwareaufsätze heraus. Im Bereich Entwicklung kann es große Einsparungen geben dadurch, dass nur ein Teilabschnitt des zu untersuchenden Fahrzeugs gefertigt werden muss und dass die einfache Bedienung es praktisch jedem ermöglicht, den Entwicklungsprozess erlebend zu unterstützen. Kritisch angesehen wurden vor allem die erwarteten hohen Kosten, die mit der Anschaffung des Systems einhergehen und ein hoher Aufwand, bei der Anpassung des Systems an neue Modelle. Des Weiteren ist der Sicherheitsaspekt ein ungewisser Punkt, da bei Benutzen des Head Mounted Display der Roboter und eventuell gefährdend geringe Abstände zu diesem nicht rechtzeitig wahrgenommen werden können. In Abstraktion an den voraussichtlichen Einsatzzweck wurde die Möglichkeit des ÜberKopf-Arbeitens und ein allgemein größerer Arbeitsraum in Frage gestellt. Zuletzt wurde der Stand der Technik, speziell die Genauigkeit des Handtrackings und die grafische Darstellung genannt. Ein Punkt, der allgemein als Schwierigkeit angesehen wurde ist, dass die Skepsis vor allem gegenüber Robotern im Allgemeinen zu merken ist. „Es muss ein Benefit erkennbar sein und eine Verbesserung für den Arbeiter sein, keine Abnahme seiner Arbeit.“ führt ein Teilnehmer aus. Die Akzeptanz ist bei der Einführung neuer Technologien sehr wichtig aber schwer, da „handwerkliche Tätigkeiten meist durch Roboter abgebaut werden. Ihre Unterstützung soll aber Effizienz fördern und nicht Arbeitskräfte ersetzen" so Teilnehmer \#1.

\section{Bewertung der Faktoren}

Aus der Bewertung der einzelnen Faktoren hat sich ergeben, dass fast alle Faktoren mit Werten über 2,5, also der Hälfte der zu vergebenen Punkte bewertet wurden (siehe Abbildung 2). Am stärksten wurde die Priorität des Faktors Sicherheit mit einem Mittelwert von 4,6 bewertet. Die beiden folgenden Faktoren sind die Modularität und Ergonomie. Der Faktor mit den geringsten Werten hat die Ortsunabhängigkeit des Systems. Darüber stehen die Schulungsnotwendigkeit und die Größe des hybriden Prototyps. 
Zur Überprüfung, ob sich der niedrigste und höchst bewertete Faktor in ihrer Wichtigkeit/Priorisierung voneinander unterscheiden, wurde ein T-Test der Bewertungen der Sicherheit und Ortsunabhängigkeit gerechnet. Diese unterscheiden sich signifikant $(p<0,001)$.

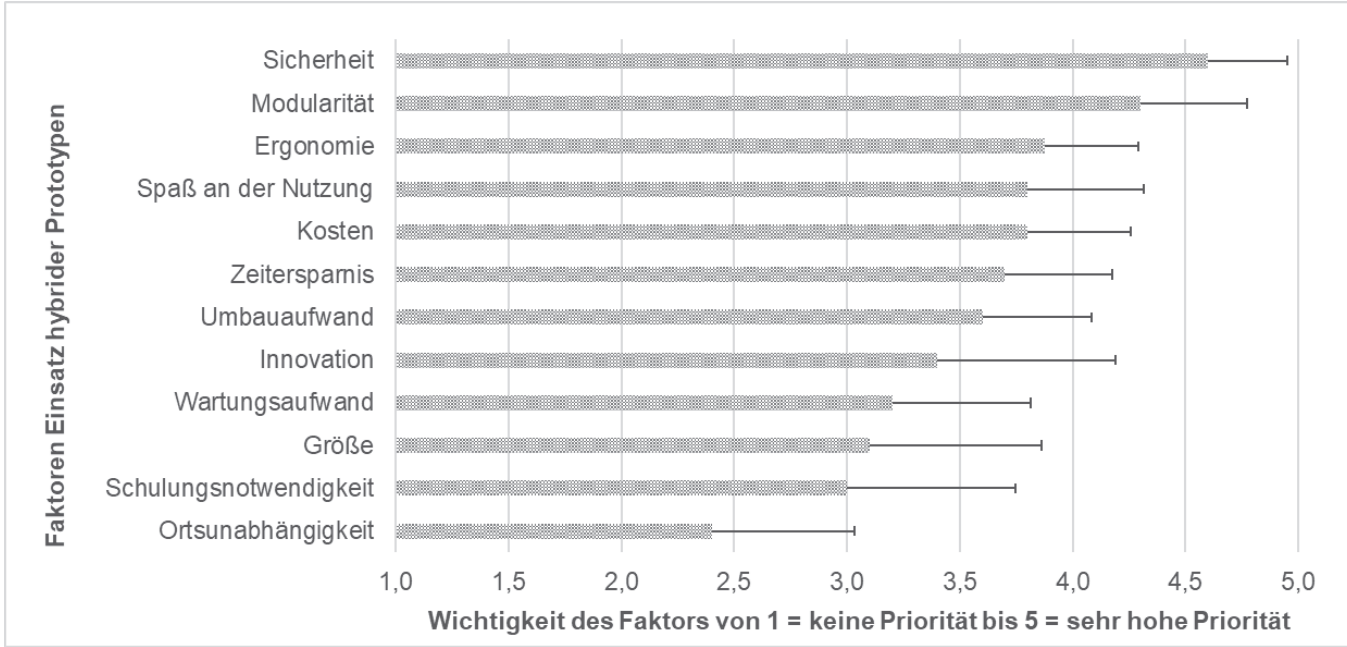

Abbildung 2: Mittelwerte und Standardabweichung der Faktorenbewertung

\section{Anwendungsfälle}

Die Nachfrage nach möglichen Anwendungsgebieten ergab ein sehr breites Feld an Ideen, da „alles, was der Mensch bewegt“ prinzipiell abgebildet werden kann. Begonnen im Bereich Montage wurden Montagetraining und Schulungen genannt, Montagetests, ob ein Werkstück oder Werkzeug platzierbar ist und Montageplanung im Sinne der Erreichbarkeit von Teilen oder ergonomischer Arbeitsplatzbewertung. Im Bereich der Qualitätsuntersuchung können beispielsweise Bedienkräfte von Schaltern oder Einrastrückmeldung messbar und gleichzeitig erlebbar gemacht werden. Außerdem kann die Zusammenarbeit zwischen Entwicklung und Konstruktion am hybriden Prototyp vereinfacht werden, da Änderungen sofort sichtbar und erlebbar gemacht werden und vor Ort diskutiert werden können.

Abseits der Industrieanwendungen wurden außerdem Arbeitsplatzuntersuchungen für körperlich eingeschränkte oder ältere Personen in Alltagssituationen genannt oder Training von Bewegungsabläufen, wie der Aufschlag bei Tennis, im Bereich Sport oder gar Physiotherapie. 


\section{Diskussion}

\section{Sicherheit}

Der Faktor Sicherheit wurde mit einem Mittelwert von 4,6 bei einer Standardabweichung von 0,7 am höchsten bewertet. Die sich daraus ergebende Gestaltung hybrider Systeme zielt darauf ab, dass die Sicherheit und Sicherheitsmaßnahmen deutlich kommuniziert und auch am Gerät selbst angezeigt werden sollten, um die Akzeptanz wesentlich zu erhöhen. Vier der Teilnehmer betonen hierbei auch die erhöhten Sicherheitsvoraussetzungen in der Produktion, im Gegensatz zum Einsatz in der Entwicklung. Drei Teilnehmer betonen, dass Sicherheitsmaßnahmen ständig erhöht werden und sieben Teilnehmer beschrieben Formen von Einschränkungen, die zur Erfüllung der Sicherheitsanforderungen notwendig werden könnten. Wiederholte Vorschläge beinhalten Sicherheitszäune und Lichtschranken, die dafür sorgen, dass der Roboter sich nicht in ungewollte Richtungen, wie im Fall der Heckklappe etwa seitlich, bewegt und dass detektiert wird, sobald sich Personen dem Versuchsraum nähern. Aufgrund der direkten Interaktion mit dem System sollte eine Näherung aus Bedienerrichtung jedoch weiterhin gewährleistet sein. Vorstellbar war eine Lichtschranke, die die Nähe des Kopfes zur bewegten Heckklappe (zum Beispiel beim automatischen Öffnen auf Knopfdruck) erkennt. Auf der anderen Seite sind softwareseitige Einschränkungen, die in die Robotersteuerung integriert sein müssen, eine Möglichkeit maximale Kraft- oder Geschwindigkeitswerte vorzugeben. Wobei diese Einschränkung die flüssige Bedienung behindern kann (zum Beispiel beim Zuwerfen der Klappe) und gut abgestimmt sein muss. Die Akzeptanz der Nutzer setzt die Umsetzung der Schutzprinzipien der Roboter-Mensch Kollaboration voraus. Das System kann aber nur effektiv eingesetzt werden, wenn die simulierten Kräfte dem eigentlichen Verhalten des realen Fahrzeugs gleich sind. Dieser Trade-off ist als Gestaltungsanspruch an die Entwickler eine Herausforderung.

\section{Modularität und Umbauaufwand}

Die Modularität wurde mit einer Standardabweichung von 0,53 mit einem Mittelwert von 4,5 bewertet. Die Modularität des Systems stellt einen eindeutigen Vorteil in der Nutzung eines Roboters dar. „Das ist schon wichtig, ansonsten brauche ich keinen Roboter." sagt Teilnehmer \#5. „Das ist ein riesen Kaufargument.“ laut Teilnehmer \#3. Die Entwicklung hybrider Systeme sollte daher für spezifische Aufgaben konfigurierbar sein, damit der effiziente Einsatz möglich ist. Aufgrund hoher Anschaffungskosten (auf organisatorischer Ebene) muss ein solches System für die Erfüllung vielerlei Aufgaben einsetzbar sein. Würde man das System für Montagetests einsetzen, bei der die Ergonomie mituntersucht wird, so ist fraglich, ob die Situation umfassend genug abgebildet 
werden kann. Zum Beispiel ist ein Anlehnen an ein Fahrzeug, um innenliegende Teile mit einem Werkzeug zu erreichen, nicht möglich, da vom System nur ein Kontaktpunkt zum Menschen (in diesem Falle das Werkzeug) dargestellt wird, fiel Teilnehmer \#6 auf. Im Gespräch stellte sich bei vier der Teilnehmer ein direkter Zusammenhang zwischen Modularität und Umbauaufwand heraus, da eine hohe Modularität wiederum hohen Umbauaufwand bedeuten kann. Der Umbauaufwand wurde im Mittel mit 3,6 bewertet. Die Standardabweichung beträgt 0,97. Geringer Umbauaufwand stellt einen großen Mehrwert dar, denn „das sind Rüstzeiten, wenn man es aus der Produktionssicht betrachtet" so Teilnehmer \#9. Um also die Akzeptanz für hybride Systeme zu steigern muss ein Gleichgewicht zwischen umfangreichen Anwendungsmöglichkeiten bei möglichst einfacher Konfiguration entwickelt werden.

\section{Ergonomie}

Die Ergonomie wurde im Mittel mit 3,9 bei einer Standardabweichung von 0,83 bewertet. Laut Gespräch ist die Ergonomie "nicht aus den Augen zu verlieren“ so Teilnehmer \#3 und laut Teilnehmer \#8 mit der Akzeptanz zur Benutzung des Systems verknüpft.

\section{Spaß an der Nutzung}

Der Faktor Spaß an der Nutzung wurde mit einem Mittelwert von 3,8 bei einer Standardabweichung von 1,03 bewertet. Aus dem Gespräch ging hervor, dass die Nutzung zwar "dem Nutzer auch etwas bringen sollte", da die Benutzerakzeptanz gefördert wird, sagt Teilnehmer \#1, es aber gleichwohl "keine reine Spaßfrage“ ist und der Spaß an der Nutzung "eher nicht im Vordergrund steht" so Teilnehmer \#7 und \#4. Die Nutzerakzeptanz kann durch ein einfaches und angenehmes Bedienerlebnis erhöht werden.

\section{Kosten, Zeitersparnis und Wartungsaufwand}

Der Faktor Kosten wurde mit einem Mittelwert von 3,8 bei einer Standardabweichung von 0,92 bewertet. Die Kosten wurden von sechs Teilnehmern mit dem Nutzen des Systems in Verbindung gebracht. In den Fällen wo noch kein konkreter Anwendungsfall vor Augen geführt werden konnte, wurden die Kosten mit mittlerer Priorität bewertet.

Die Zeitersparnis wurde im Mittel mit 3,7 bewertet, die Standardabweichung beträgt 0,95. Die Kosten-Nutzen-Rechnung wurde von zwei Teilnehmern beim Gespräch über die Zeitersparnis wieder eingebracht. Auch hier waren fehlende konkrete Vorstellungen des Anwendungsfalles als Grund für schwere Bewertbarkeit der Priorität aufgeführt. 
Der Faktor Wartungsaufwand wurde mit einem Mittelwert von 3,2 bei einer Standardabweichung von 1,23 bewertet. Er wurde von vier Teilnehmern im direkten Zusammenhang zu den Kosten genannt und von zwei Teilnehmer vom Nutzen abhängig gemacht. Der Einsatz des Systems in der Entwicklung lässt Wartungszeiträume jedoch leichter zu als ein Einsatz in der Produktion, so Teilnehmer \#8. Um die Akzeptanz für hybride Systeme zu steigern, ist es sinnvoll, Kosten-Nutzen-Rechnungen für konkrete Anwendungsfälle zu präsentieren.

\section{Innovation}

Der Faktor Innovation wurde mit einem Mittelwert von 3,4 bewertet. Die Standardabweichung liegt bei 1,58, was auch in den Gesprächen widergespiegelt wurde. Auf der einen Seite ist Innovation „ein riesen Kaufargument" (Teilnehmer \#3) und „das wichtigste dabei“ (Teilnehmer \#9), auf der anderen Seite ist es "nicht ausschlaggebend“ (Teilnehmer \#5) und "nicht der Grund, eine solche Beschaffung anzustoßen“ (Teilnehmer \#10). Die Innovation des Systems hat das Potential die Akzeptanz zu fördern.

\section{Größe}

Der Faktor Größe wurde mit einem Mittelwert von 3,1 bei einer Standardabweichung von 1,52 bewertet. Im Gespräch ist aufgefallen, dass der Anspruch an die Größe des Systems abhängig ist, vom Platz, der zur Verfügung steht, welcher von Firma zu Firma stark variiert. Für Teilnehmer \#2 sind die Außenmaße des Systems nicht so relevant, wie der nutzbare Arbeitsraum, für Teilnehmer \#9 sollte das System „so klein wie möglich" sein. Der Arbeitsraum sollte in Relation zur Gesamtgröße des Systems möglichst groß sein.

\section{Schulungsnotwendigkeit}

Der Faktor Schulungsnotwendigkeit wurde mit einem Mittelwert von 3,0 bei einer Standardabweichung von 1,49 bewertet. Schulungsnotwendigkeit wird erwartet, aber auch in Kauf genommen, so drei der Teilnehmer, da eine externe Instanz zur Anpassung des Geräts auf neue Praxisanwendungen als nicht sinnvoll angesehen wird.

\section{Ortsunabhängigkeit}

Die Ortsunabhängigkeit wurde im Mittel mit 2,4 bewertet. Die Standardabweichung beträgt 1,26. Die Antworten weichen sehr stark voneinander ab, abhängig vom hypothetischen Anwendungsfall. Auf der einen Seite würde nach langfristiger und ausgiebiger Planungsphase zur Anschaffung des Gerätes das Gerät an einem Ort verbleiben, 
so Teilnehmer \#10. Vor allem im Hinblick auf die Absicherung gegen Umfallen (Verschraubung im Boden) und Sicherheitsmaßnahmen wie Lichtschranken oder eine Einhausung wäre ein späteres Verlagern nicht vorgesehen, beschreibt Teilnehmer \#9. Auf der anderen Seite wird das Remote Arbeiten als interessante Möglichkeit angesehen, die jedoch keinen Einfluss auf eine höhere Priorisierung hat. Da die Arbeit mit dem System eine direkte Interaktion erfordert, wären für die Arbeit von der Ferne zwei identische Systeme für die gemeinsame Entwicklung erforderlich. Das Einspeisen von neuen Versuchsdaten/ -Modellen aus der Ferne wäre möglich, jedoch geht der Vorteil des sofortigen haptischen Erlebnisses verloren, erläutert Teilnehmer \#3. Ein Konzept zum Remote Arbeiten kann die Akzeptanz des Systems erhöhen.

\section{Ausblick}

Es ist geplant, Usability Tests mit Nutzern am oben besprochenen hybriden Prototyp durchzuführen. Anschließend können die Faktoren von den Studienteilnehmern bewertet werden, wodurch die durch die Bedienung gewonnenen Eindrücke mit der Studie verglichen werden können. Dies würde die Frage untersuchen, ob und inwiefern sich die Faktoren für die Nutzung in ihrer Bedeutsamkeit durch die Bedienung ändern.

\section{Danksagung}

Das KMU-innovativ-Verbundprojekt „ROBVRAR: Funktionale virtuelle Prüfsysteme für roboterbasierte Qualitätsprüfung" wurde gefördert durch das BMBF, Förderkennzeichen $01 \mid$ S18015B

\section{Literaturverzeichnis}

Becker, Waldemar; Biedermann, Niels; Blume, Claas; Geiger, Andreas; Hertzer, Maximilian; Kind, Simon et al. (2018): VIBSHP. Virtuelle Inbetriebnahme für Industrie 4.0 zukunftssicher beherrschen: modulare Gestaltung und immersive, digitale Absicherung von mechatronischen Produktionsanlagen. Hg. v. Rainer Stark, Patrick Müller und Hendrik Grosser. Stuttgart: Fraunhofer Verlag.

Bermejo, Carlos; Hui, Pan (2017): A survey on haptic technologies for mobile augmented reality, zuletzt geprüft am 25.03.2021.

Bowman, Doug A. (2005): 3D user interfaces. Theory and practice. Boston: Addison-Wesley. Online verfügbar unter http://proquest.tech.safaribooksonline.de/9780133390599, zuletzt geprüft am 25.03.2021. 
Brandenburg, Elisabeth (2020): Gestaltungsrichtlinien für die Visualisierung von Produktinformationen in der virtuellen Umgebung CAVE zur Unterstützung von Design Review Teams. Stuttgart: Fraunhofer Verlag (Berichte aus dem Produktionstechnischen Zentrum Berlin).

DIN EN ISO 9241-11: Ergonomische Anforderungen für Bürotätigkeiten mit Bildschirmgeräten - Teil 11: Anforderungen an die Gebrauchstauglichkeit - Leitsätze (ISO 9241-11:1998).

Grajewski, Damian; Górski, Filip; Hamrol, Adam; Zawadzki, Przemysław (2015): Immersive and Haptic Educational Simulations of Assembly Workplace Conditions. In: Procedia Computer Science 75 (1), S. 359-368. D0I: 10.1016/j.procs.2015.12.258.

Stark, Rainer; Beckmann-Dobrev, Boris; Schulze, Ernst-Eckart; Adenauer, Julian; Israel, Johann Habakuk (2009): Beiträge 8. Berliner Werkstatt MMS 2009.

van der Laan, Jinke D.; Heino, Adriaan; Waard, Dick de (1997): A simple procedure for the assessment of acceptance of advanced transport telematics. In: Transportation Research Part C: Emerging Technologies 5 (1), S. 1-10. D0I: 10.1016/S0968090X(96)00025-3.

ISO/TS 15066: VDMA Positionspapier MRK-Sicherheit.

Volkov, Sergei; Vance, Judy M. (2001): Effectiveness of Haptic Sensation for the Evaluation of Virtual Prototypes. In: Journal of Computing and Information Science in Engineering 1 (2), S. 123-128. D0I: 10.1115/1.1384566.

\section{Kontakt}

Liesa Zimmermann

Prof. Dr.-Ing. Rainer Stark

Fachgebiet Industrielle Informationstechnik

Technische Universität Berlin

Sekretariat PTZ4

Pascalstraße 8-9

10587 Berlin

www.iit.tu-berlin.de

Kathrin Konkol

Dr. Elisabeth Brandenburg

Fraunhofer IPK

Pascalstraße 8-9

10587 Berlin

ipk.fraunhofer.de 